\title{
Study on the Optimal Number of Transducers for Pipe Flow Rate Measurement Downstream of a Single Elbow Using the Ultrasonic Velocity Profile Method
}

\author{
Sanehiro Wada, ${ }^{1}$ Kenichi Tezuka, ${ }^{1}$ Weerachon Treenuson, ${ }^{2}$ \\ Nobuyushi Tsuzuki, ${ }^{2}$ and Hiroshige Kikura ${ }^{2}$ \\ ${ }^{1}$ Engineering R\&D Division, Tokyo Electric Power Company, 4-1, Egasaki-cho, Tsurumi-ku, Yokohama 230-8510, Japan \\ ${ }^{2}$ Research Laboratory for Nuclear Reactors, Tokyo Institute of Technology, 2-12-1, Ohokayama, Meguro-ku, Tokyo 152-8550, Japan \\ Correspondence should be addressed to Sanehiro Wada, wada.sanehiro@tepco.co.jp
}

Received 15 June 2012; Revised 27 September 2012; Accepted 22 October 2012

Academic Editor: Yan Yang

Copyright (๑) 2012 Sanehiro Wada et al. This is an open access article distributed under the Creative Commons Attribution License, which permits unrestricted use, distribution, and reproduction in any medium, provided the original work is properly cited.

This paper presents a new estimation method to determine the optimal number of transducers using an Ultrasonic Velocity Profile (UVP) for accurate flow rate measurement downstream of a single elbow. Since UVP can measure velocity profiles over a pipe diameter and calculate the flow rate by integrating these velocity profiles, it is also expected to obtain an accurate flow rate using multiple transducers under nondeveloped flow conditions formed downstream of an elbow. The new estimation method employs a wave number of velocity profile fluctuations along a circle on a pipe cross-section using Fast Fourier Transform (FFT). The optimal number of transducers is estimated based on the sampling theorem. To evaluate this method, a preliminary experiment and numerical simulations using Computational Fluid Dynamics (CFD) are conducted. The evaluating regions of velocity profiles are located at 3 times of a pipe diameter $(3 D)$ for the experiment, and 1 and $5 D$ for the simulations downstream of an elbow, respectively. Reynolds numbers for the experiment and simulations are set at $4 \times 10^{4}$ and $5 \times 10^{6}$, respectively. These results indicate the efficiency of this new method.

\section{Introduction}

Accurate pipe flow rate measurements are important to generate electricity with high efficiency at nuclear power plants. Highly accurate flow metering has also been required recently to improve plant performance and reduce the environmental impact of plant discharge on the surrounding sensitive environment. The accuracy of nuclear reactor feed water flow rate measurements is receiving special attention, since they represent the largest source of uncertainty when calculating reactor thermal power. The accuracy of flow rate measurement for turbine cooling systems is also important to monitor any temperature increase in discharge water and improve steam condenser performance. We aim to apply the UVP method to measure the flow rate of feed and circulation water in power plants.

Compact piping configurations are often used at the suction of centrifugal pumps due to space restrictions.
The generation of secondary flow structures such as swirls, asymmetry, and flow distortion within such systems is strongly dependent on the piping system geometry, for example, the angle of pipe bends, bend radii, planes of curvature, types of elbows, and lengths between elbows.

The UVP method allows instantaneous velocity profiles to be obtained along the ultrasonic beam path. The principle of the flow measurement method is based on integrating an instantaneous velocity profile over a pipe diameter. This method can obtain the velocity component along the ultrasonic beam, while the streamwise velocity component can be calculated provided the pipe flow is fully developed with long straight pipe sections upstream of this meter.

To validate this technique and investigate its accuracy, calibration tests were performed at the National Institute of Standards and Technology (NIST) flow standards located in Gaithersburg, MD, USA $[1,2]$. These results showed that the difference between the average flow rate, as measured by 


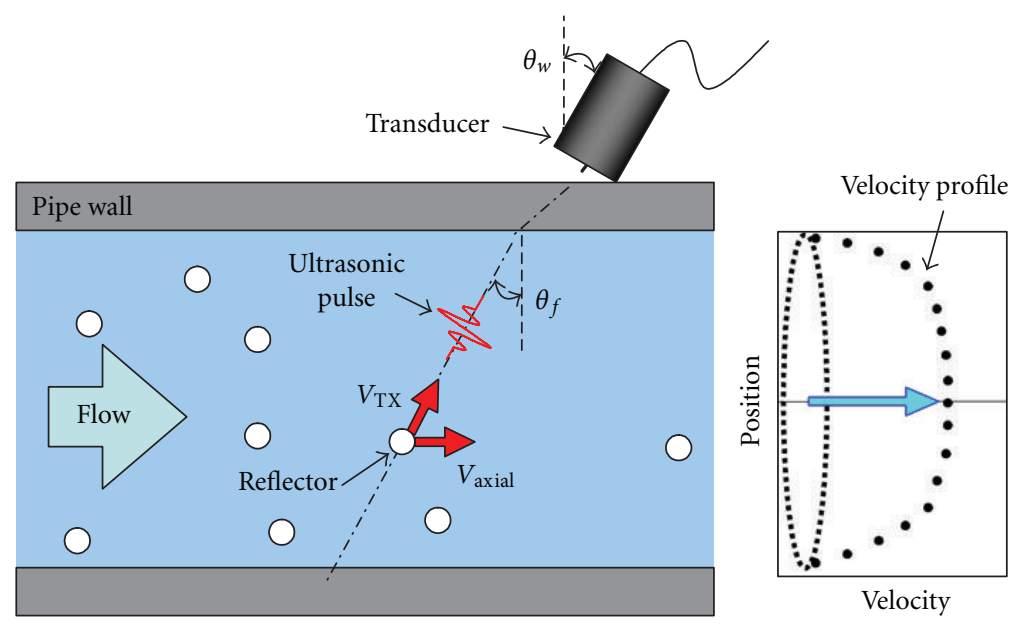

FIgURE 1: Ultrasonic propagation in the UVP method.

the UVP method and NIST gravimetric measurement, was approximately $0.18 \%$.

To evaluate the effect of pipe surface roughness on the accuracy of the meter factor using the UVP method, experiments were conducted [3]. For nondeveloped flows downstream of a single elbow, the multimeasuring line method was applied [4]. This method employed three transducers mounted on a pipe with a constant circumferential interval. However, since these experiments were conducted 8 diameters downstream of a single elbow, the secondary flow influence was relatively minor in comparative terms compared with the case of 1 or 2 diameters downstream of a single elbow. Power plants often require accurate flow rate monitoring, even without sufficient straight pipe sections upstream of the measuring point. Particularly for large diameter pipes, it is difficult to arrange a sufficient straight pipe section upstream of the measuring point and the distance between a single elbow and the measuring point is often only a few diameters. To apply UVP to accurate flow rate measurement under this condition, estimating the optimal number of transducers is important with costs in mind.

Based on conventional studies, secondary flow structures downstream of a single elbow were reported [5-8], and the effects of a single elbow on flow rate measurement accuracy were investigated using an orifice flow meter [9]. However, few investigations have been conducted on flow rate measurements under the condition of a strong secondary flow a few diameters downstream of a single elbow.

To measure flow accurately under such condition, detailed knowledge of the flow field downstream of a single elbow is required to minimize the adverse effects of secondary flows. Such knowledge can be effectively and efficiently gained using CFD.

This paper presents a new estimation method to determine the optimal number of transducers to measure flow rate accurately downstream of a single elbow. To evaluate this new method, a preliminary experiment was conducted under conditions of a Reynolds number of $\operatorname{Re}=4 \times 10^{4}$, a pipe inner diameter of $D=50 \mathrm{~mm}$, and a measuring point located at $3 D$ distance downstream of an elbow. Subsequently, CFD calculations were also conducted under the following conditions: flow rates were estimated at 1 and 5 diameters downstream of a single elbow. The inflow profiles showed fully developed turbulent pipe flow and the diameter was assumed to be $600 \mathrm{~mm}$ piping with short elbow. The Reynolds number was set at $5 \times 10^{6}$ considering the actual plant condition.

\section{Flow Rate Measurement Method Using UVP}

A UVP method for flow metering systems was developed $[10,11]$. Figure 1 shows a diagram of the ultrasound propagation involved in the UVP method, which allows instantaneous velocity profiles to be obtained along the ultrasonic beam path. The principle of the flow measurement method is based on the integration of an instantaneous velocity profile over a pipe diameter.

Ultrasound at frequency $f_{0}$ is emitted into the fluid at an incident angle $\theta_{w}$ from a transducer. The ultrasonic pulses bounce back from particles flowing in the fluid with the frequency shifted $f_{d}$ based on the Doppler principle, which is propositional to the particle velocity $V_{\mathrm{TX}}$. By assuming a one-directional flow parallel to the streamwise direction for a fully developed flow, the velocity in the axial direction $V_{\text {axial }}$ can be obtained as follows:

$$
V_{\text {axial }}=\frac{V_{\mathrm{TX}}}{\sin \theta_{f}},
$$

where $\theta_{f}$ is the transmission angle of ultrasound in the fluid. The particle position $L_{f}$ is also determined along the ultrasound path. By measuring the traveling time of the ultrasonic pulse $t_{f}$ for the round trip between the transducer and the targeting particle in the fluid, the particle position $L_{f}$ can be calculated as the distance from the transducer to the particle at the speed of the ultrasound in the fluid. 


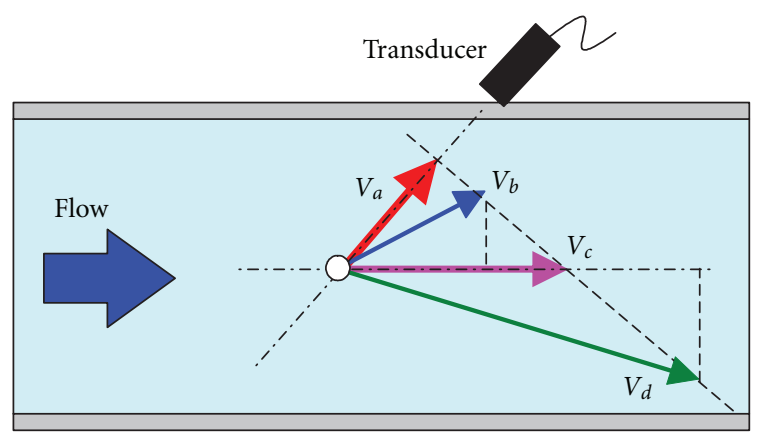

FIGURE 2: Velocity component of tracer particles flowing in fluid obtained by the UVP method.

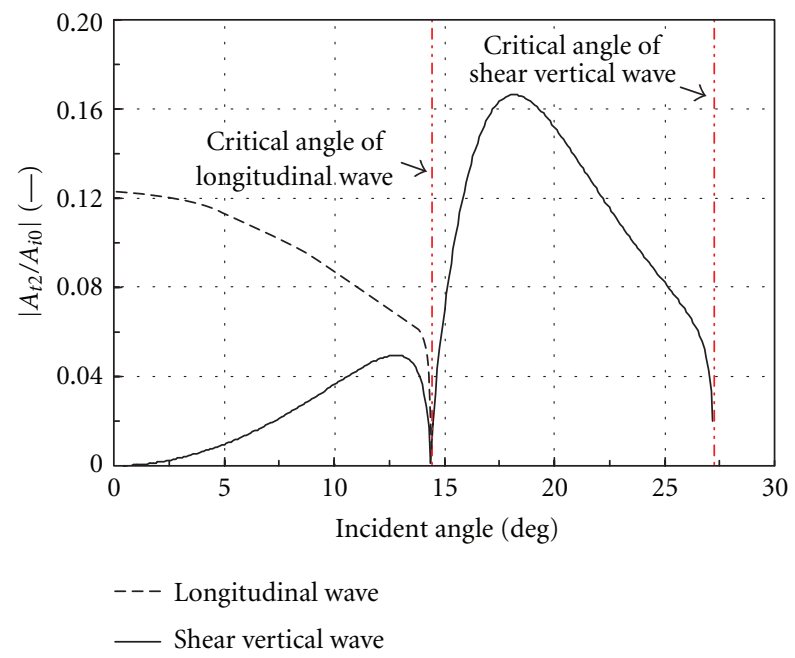

FIgURE 3: Transmission ratio of ultrasound from carbon steel to water versus the incident angle of ultrasound wave into carbon steel plate. $\left(A_{i 0}\right.$ : amplitude of incidence ultrasound, $A_{t 2}$ : amplitude of transmission).

The following equations show the relationships used in the UVP method:

$$
\begin{aligned}
V_{\mathrm{TX}} & =\frac{c_{f}}{2 f_{0}} f_{d}, \\
L_{f} & =\frac{c_{f} t_{f}}{2} .
\end{aligned}
$$

The flow conditions downstream of elbows are usually not fully developed flow due to the strong secondary flow. Therefore, velocity profiles obtained by UVP are influenced by the secondary flow. For example, as illustrated in Figure 2, while the actual velocity vector varies from $V_{b}$ to $V_{d}$, the velocity component obtained using UVP is the same value, $V_{a}$.

Studies on ultrasonic transmission efficiency through various media are needed to consider accurate flow rate measurements. Figure 3 shows an ultrasonic transmission ratio where the ultrasound enters water through a carbon

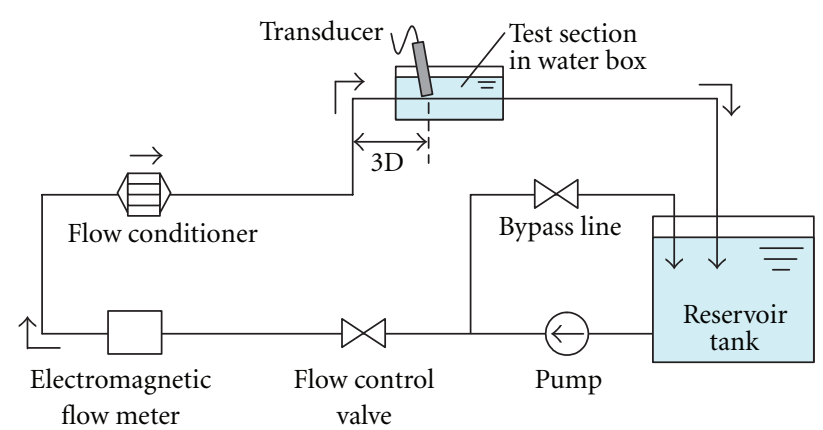

FIgURE 4: Schematic diagram for the preliminary experiment.

steel pipe wall. The transmission ratio can be calculated as follows [12]:

$$
\begin{gathered}
\frac{A_{t 2}}{A_{i 0}}=\frac{4 \lambda_{0} \mu_{1} l_{0} l_{1}\left(1+l_{0}\right)\left(s_{1}^{2}-1\right)\left(s_{1}^{4}-1\right)}{F_{1} F_{2}}, \\
F_{1}=\lambda_{0} l_{1}\left(1+l_{0}^{2}\right)\left(1+s_{1}^{2}\right)+\mu_{1} l_{0}\left\{\left(s_{1}^{2}-1\right)^{2}+4 l_{1} s_{1}\right\}, \\
F_{2}=\lambda_{2} l_{1}\left(1+l_{2}^{2}\right)\left(1+s_{1}^{2}\right)+\mu_{1} l_{2}\left\{\left(s_{1}^{2}-1\right)^{2}+4 l_{1} s_{1}\right\}, \\
l_{0}=\cot \theta_{l 0}, \\
l_{1}=\cot \theta_{l 1}, \\
s_{0}=\cot \theta_{s 0}, \\
s_{1}=\cot \theta_{s 1} .
\end{gathered}
$$

$A$ is the amplitude of the ultrasound pulse, $\lambda$ and $\mu$ are the Lame constant of the medium, and $\theta$ is the incident or refraction angle of the ultrasound pulse.

The propagation and transmission of ultrasound follow the wave propagation theorem. Reflection, diffraction, and inflection of ultrasound pulses occur at the boundary of two materials with different acoustic impedances. In solids, both longitudinal and shear vertical waves are generated with different refraction angles, the transmission ratio of which can be estimated considering the ultrasound incident angle and the acoustic parameters of these materials. Multiple wave paths emerge in the flow field, which affect the accuracy of flow rate measurement [13]. Therefore, to measure the flow rate in a pipe through the pipe outer wall, adopting the shear vertical wave is preferable because the transmission wave into water forms a strong and single wave path. Since the transmission angle in water is approximately $18^{\circ}$, as illustrated in Figure 3 when adopting the shear vertical wave, the secondary flow has a remarkable effect on velocity profiles obtained by the UVP method.

The UVP method can measure an instantaneous velocity profile as mentioned above, and the sampling rate is approximately 50 to $100 \mathrm{~ms}$ per profile when a pipe diameter is $500 \mathrm{~mm}$. Additionally, a flow rate is generally calculated using a time-averaged velocity profile averaged using over 1000 instantaneous velocity profiles. Considering these parameters, the UVP method is applicable to fluctuating flows required over a 50 to $100 \mathrm{~ms}$ sampling rate 


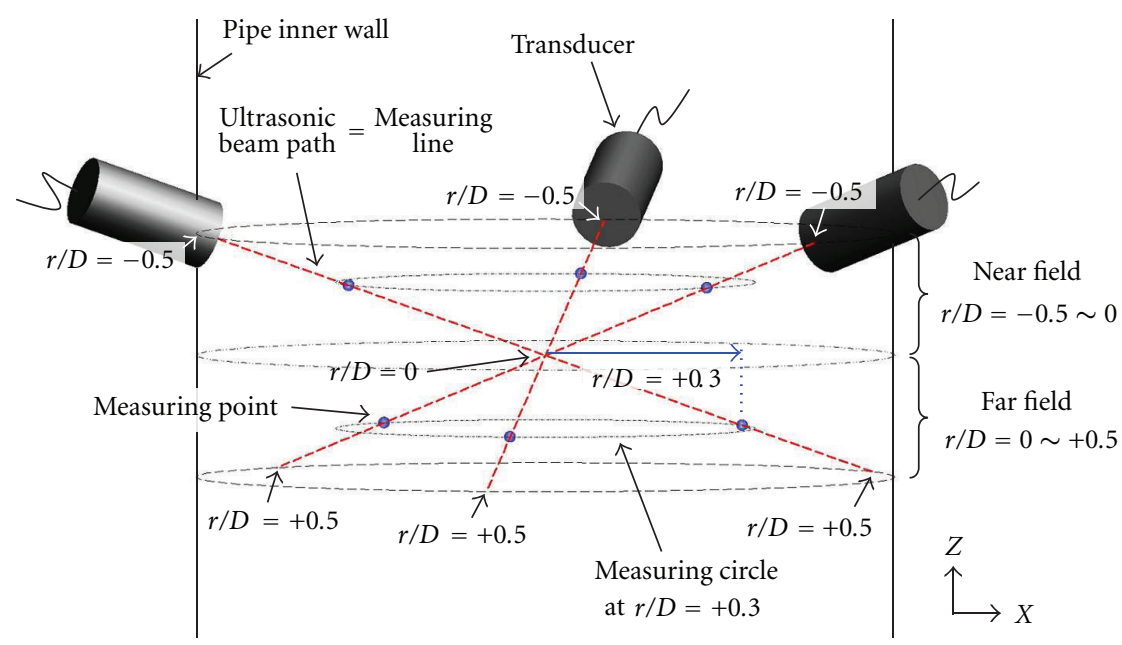

FIGURE 5: Measuring lines and circles of velocity profiles when using three transducers.

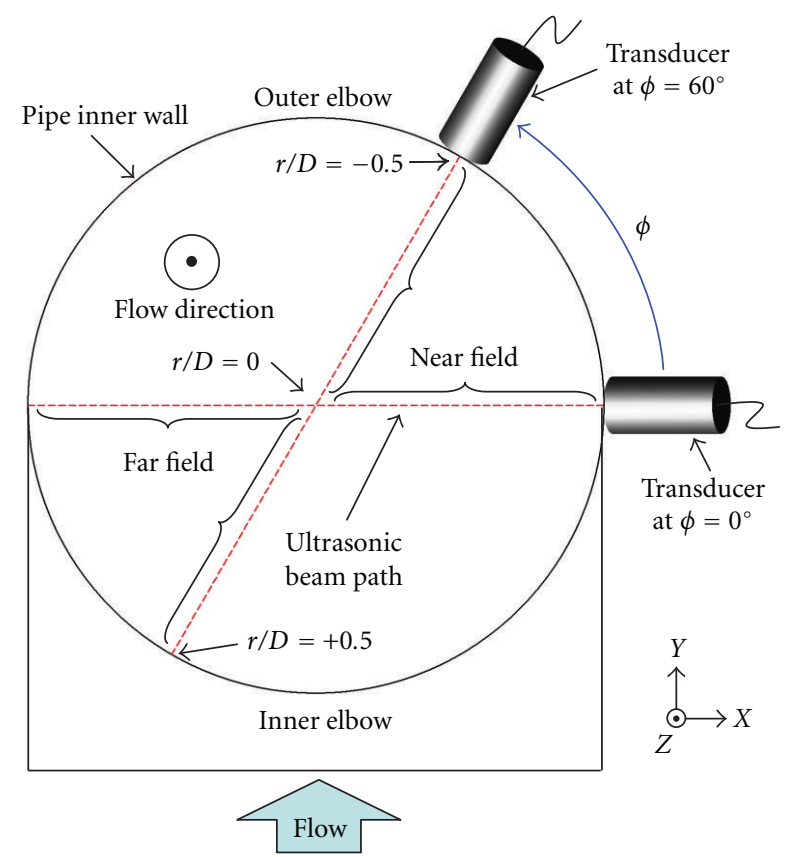

Figure 6: Circumferential transducer position $\phi$ at each crosssection plane downstream of a single elbow (the view from downstream).

(or below 10 to $20 \mathrm{~Hz}$ ). Conversely, a conventional study reported a frequency of pressure fluctuation just after an elbow of approximately 3 to $10 \mathrm{~Hz}$ for $\mathrm{Re}=10^{6}$ [14]. Therefore, in this paper, a time-averaged velocity profile is employed to investigate the optimal number of transducers for accurate flow rate metering.

\section{Preliminary Experiment}

3.1. Experimental Conditions. To evaluate the new estimation method to determine the optimal number of transducers to measure flow rate accurately downstream of a

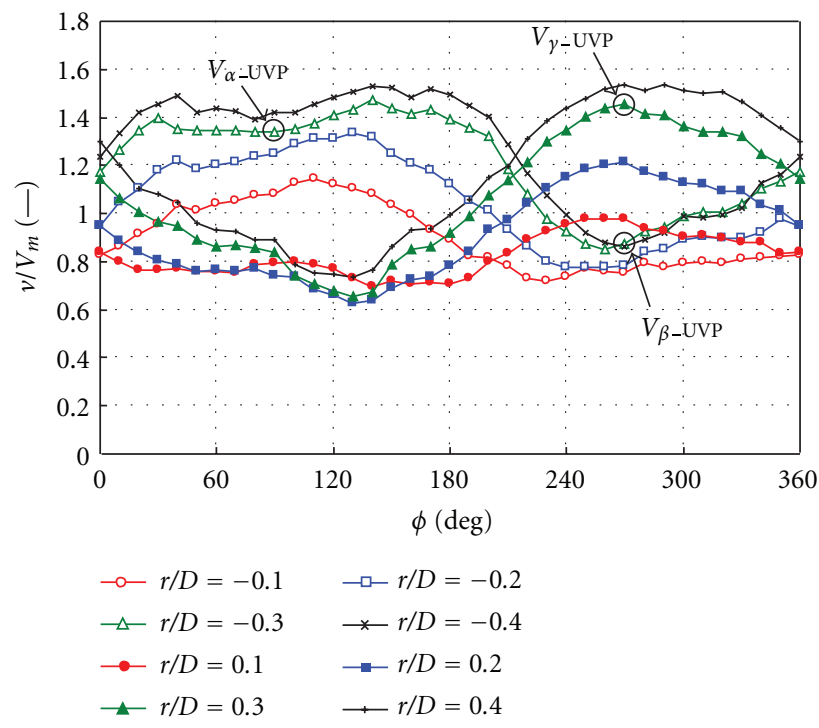

FIGURE 7: Velocity profiles along the measuring circle $\left(\mathrm{Re}=4 \times 10^{4}\right.$, $\left.L_{d}=3 D\right)$.

single elbow, a preliminary experiment was conducted. The experimental apparatus used consisted of a water circulation system, a test section, and a measurement system as shown in Figure 4 [15]. The schematic diagram was designed to emphasize the formation of fully developed turbulent pipe flow downstream of elbows. The flow rate could be controlled via a bypass line and flow control valve. Water was circulated by a centrifugal pump. The pipeline system was made of Polyvinyl Chloride (PVC). Before the test section, a flow conditioner and turbulence promoter ring were installed to ensure a uniform velocity profile.

Water was filled in a water box coupled between the transducer and pipe. The pipe at the test section was made of acrylic considering the high ultrasound transmission ratio. The total length of pipe was approximately $1.5 \mathrm{~m}$, with inner 


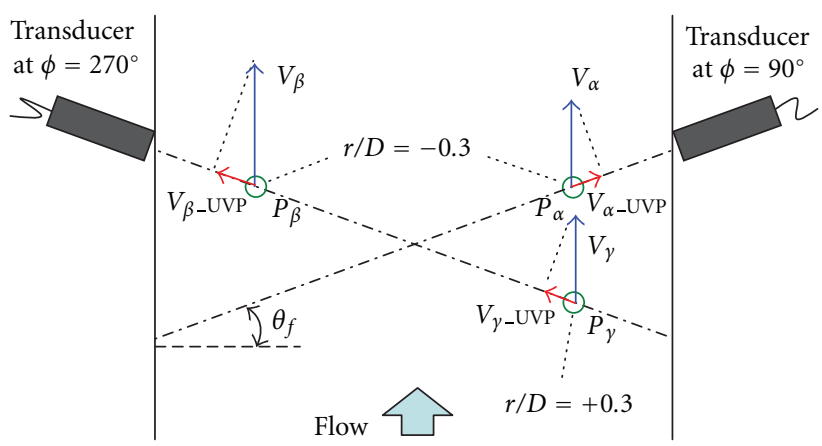

(a) Without a secondary flow

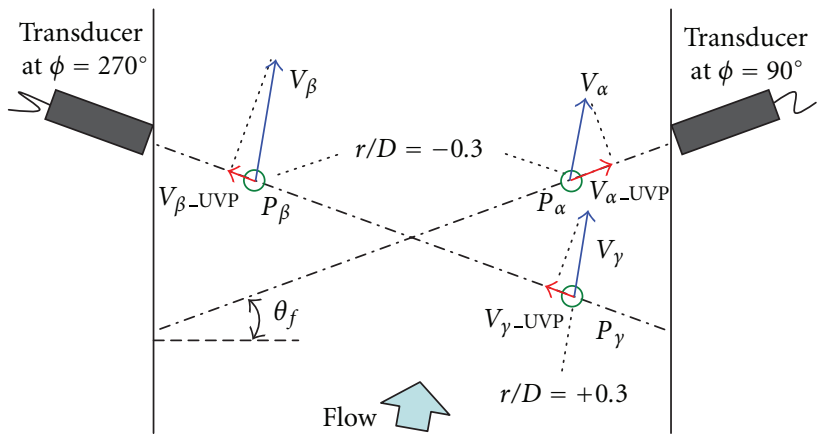

(b) With a secondary flow

FIGURE 8: Schematic diagram of the velocity component measured by the UVP method.

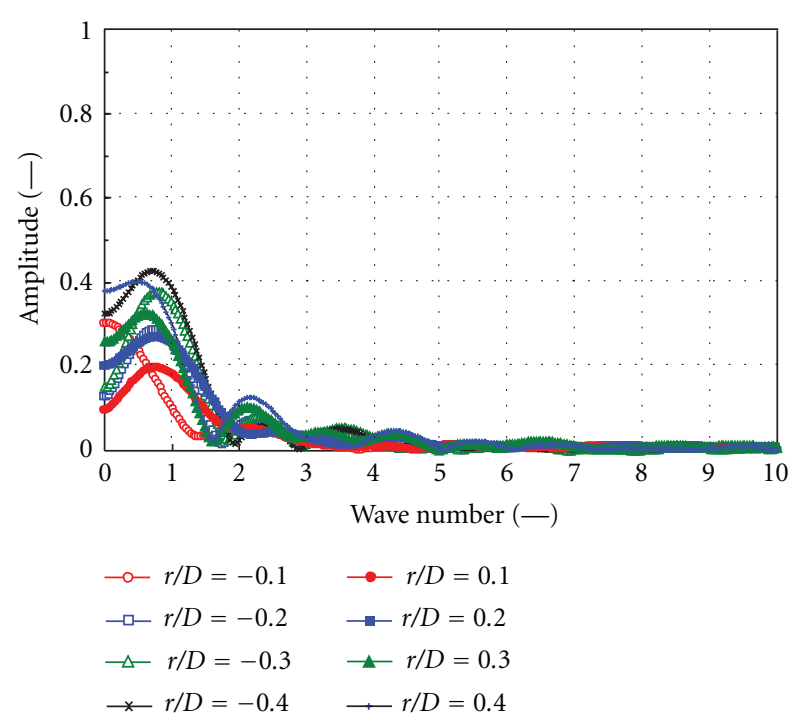

FIGURE 9: Fourier amplitude of velocity profile along the measuring circle.

diameter and wall thickness of 50 and $5 \mathrm{~mm}$, respectively. The flow rate was monitored by an electromagnetic flow meter located upstream of the test section. Elbows had curvature angles of $90^{\circ}$ and average curvature of $30 \mathrm{~mm}$. The measuring point was located at $3 D$ downstream of the elbow.

Figure 5 shows the schematic diagram of a measuring section when using three transducers. The transmission point of the ultrasound into fluid, the center point of the pipe, and the far point from the transducer on the pipe inner surface along the ultrasonic beam path are defined as $r / D=-0.5,0$ and +0.5 , respectively. A line segment from $r / D=-0.5$, to 0 (or 0 to +0.5 ) along the ultrasonic beam path and a circle passing through the measuring points on the same cross-section are defined as "measuring line" and "measuring circle," respectively. Therefore, the total number of measuring lines is six when using three transducers. The regions from $r / D=-0.5$ to 0 and from 0 to +0.5 are defined as "near field" and "far field," respectively. Additionally, the

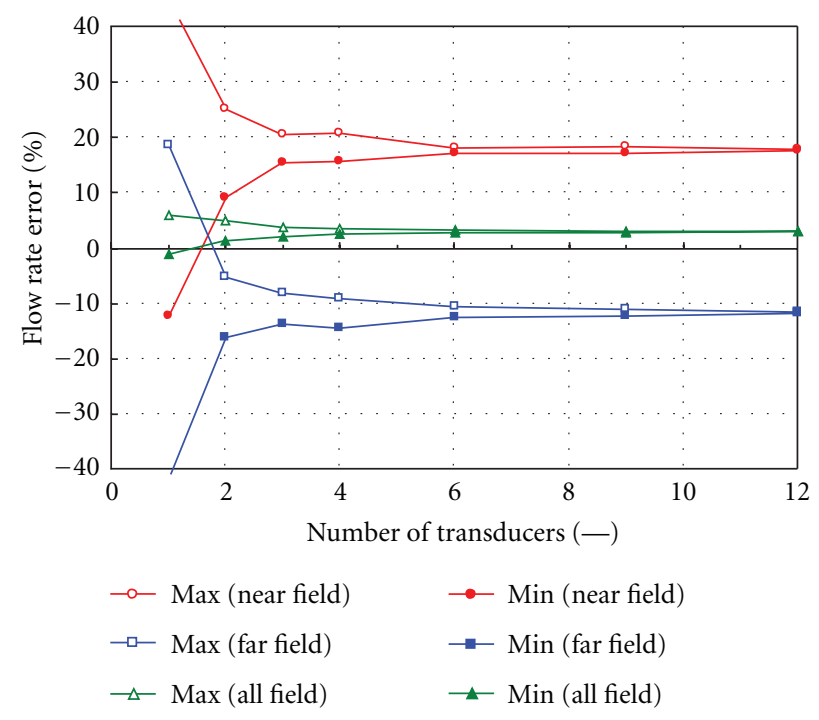

FIGURE 10: Flow rate errors versus the number of transducers.

circumferential transducer position $\phi$ on the pipe outer wall is defined as illustrated in Figure 6.

The flow measurement system is composed of the UVPDUO model (Met Flow AG) and a PC, which records the flow rate obtained by the electromagnetic flow meter and the temperature data by a thermometer. The basic ultrasound frequency is $8 \mathrm{MHz}$ and the distance between measuring points along an ultrasonic beam is $0.74 \mathrm{~mm}$. Nylon powder, the average particle diameter of which is approximately $80 \mu \mathrm{m}$ and specific particle gravity of which is 1.02 , was dispersed in water as an ultrasonic reflector. The Reynolds number is $\mathrm{Re}=4 \times 10^{4}$. During the experiment, the water temperature was kept at a constant $25^{\circ} \mathrm{C}$ using a subcooler installed inside a reservoir tank.

A time-averaged velocity profile is averaged using 1024 instantaneous velocity profiles and normalized by the mean velocity. A transducer is mounted on the outer surface of the pipe at an incident angle of $10^{\circ}$ to the normal plane of the wall. The transducer is set at $10^{\circ}$ intervals around the pipe and the velocity profiles were measured at each circumferential position. 


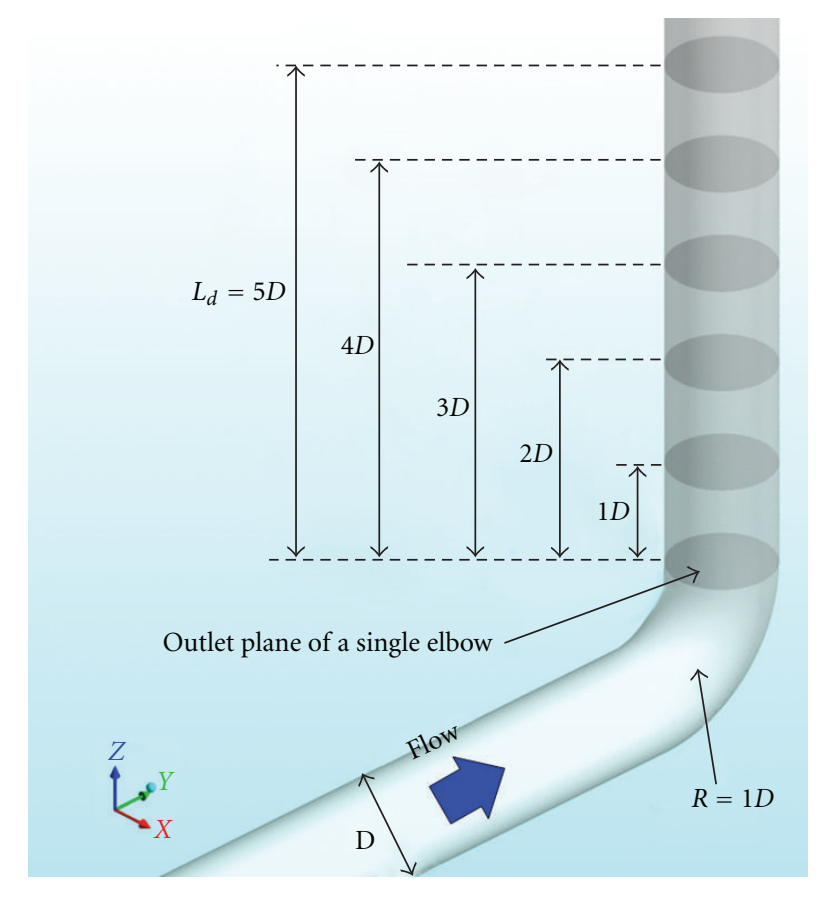

FIGURE 11: Schematic diagram of a single elbow and cross-section planes as estimating secondary flow.

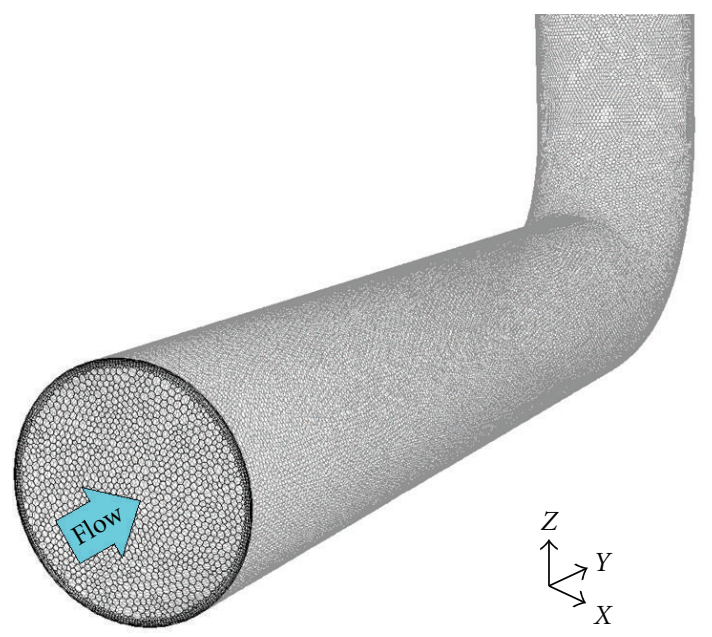

FIGURE 12: Schematic diagram of the mesh layout around the single elbow.

3.2. Results. Figure 7 shows velocity profiles along each measuring circle obtained by the UVP method. The horizontal axis is the transducer circumferential position, while the vertical axis is the time-averaged velocity normalized by the mean velocity. This figure indicates that velocity profiles fluctuate depending on the circumferential transducer position. The flow condition formed in this experiment was expected to be an asymmetrical flow along a line through $\phi=90$ and $270^{\circ}$ with small secondary flows. This is because considering velocities obtained at three points $\left(P_{\alpha}, P_{\beta}\right.$, and $\left.P_{\gamma}\right)$ as illustrated in Figure 8(a), the velocities $V_{\alpha_{-} U V P}$ and $V_{\beta_{-} \text {UVP }}$ obtained at $P_{\alpha}$ and $P_{\beta}$ differed under an asymmetric flow, velocities $V_{\alpha_{-} \text {UVP }}$ and $V_{\gamma_{-} \text {UVP }}$ obtained at $P_{\alpha}$ and $P_{\gamma}$ were almost the same without a secondary flow, due to the proximity of $P_{\alpha}$ and $P_{\gamma}$, while $V_{\alpha_{-} \text {UVP }}$ and $V_{\gamma_{-} \text {UVP }}$ differed with a secondary flow as shown in Figure 8(b). For example, when focusing on the case of $r / D=-0.3$ and +0.3 in Figure 7, $V_{\alpha_{-} \text {UVP }}$ (plotted at $\phi=90^{\circ}$ and $r / D=-0.3$ ) differed significantly from $V_{\beta_{-} \text {UVP }}$ (plotted at $\phi=270^{\circ}$ and $r / D=-0.3$ ), while $V_{\alpha_{-} \text {UVP }}$ was similar to $V_{\gamma_{-} \text {UVP }}$ (plotted at $\phi=270^{\circ}$ and $r / D=+0.3$ ). More studies regarding the effects of secondary flow on a velocity profile along a measuring circle are required for quantitative evaluation.

Investigations of the optimal number of transducers are important when flow rate measurements using the UVP method are conducted. The spatial resolution of measuring points along a measuring line can also be controlled according to the pipe diameter and the required flow rate accuracy. Conversely, the spatial resolution along a measuring circle depends on the number of transducers, the optimal number of which can be estimated by considering flow conditions, for example, swirl or asymmetric flows. Therefore, in this study, the wave number of velocity profiles fluctuation in a measuring circle was investigated using FFT analysis, and the number of transducers was also estimated using these FFT results.

Figure 9 shows the FFT results calculated using velocity profiles along measuring circles. The maximum peaks emerged at approximately $N_{w}=1$ for every $r / D$ cases, while the amplitudes declined at $N_{w}=2$ to 3 , where $N_{w}$ indicates the wave number in a measuring circle.

Flow rate errors versus the number of transducers mounted around the pipe outer wall are plotted in Figure 10. The flow rate of "All field" written in the figure is calculated using velocity profiles in both near and far fields. Since the flow is expected to vary in a streamwise direction, the converged flow rate error of the near field differs from that of far field. The flow rate errors converge when using approximately 4 to 6 transducers, and this result is consistent with the estimated number of transducers considering the sampling theorem.

\section{CFD Simulation}

4.1. CFD Code and Modeling. In this paper, CFD analysis was performed with $1 D$ mean radius elbow configuration. The CFD code used to simulate a pipe flow downstream of a single elbow was ANSYS FLUNET (Version 13.0). An isometric view of the piping configuration is shown in Figure 11. Secondary flows were estimated at six crosssections, from the outlet plane of a single elbow $(0 D)$ to $5 D$ downstream of a single elbow. Table 1 and Figure 12 show the specifications of CFD analysis and a schematic diagram of the mesh layout around the single elbow.

A velocity profile assumed to be measured by the UVP method was read out from a numerical simulation result. In this paper, the transmission angle into water was estimated at $19.4^{\circ}$ considering the material used for a pipe and wedge, which were assumed to be composed of carbon steel and polystyrene, respectively. The velocity profile was subject to change depending on the incident angle, because the 


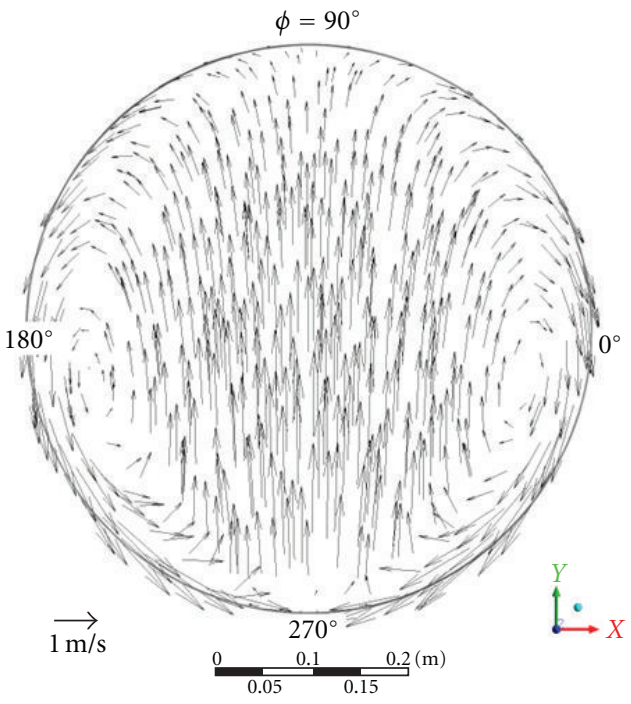

(a) $L_{d}=0 D$

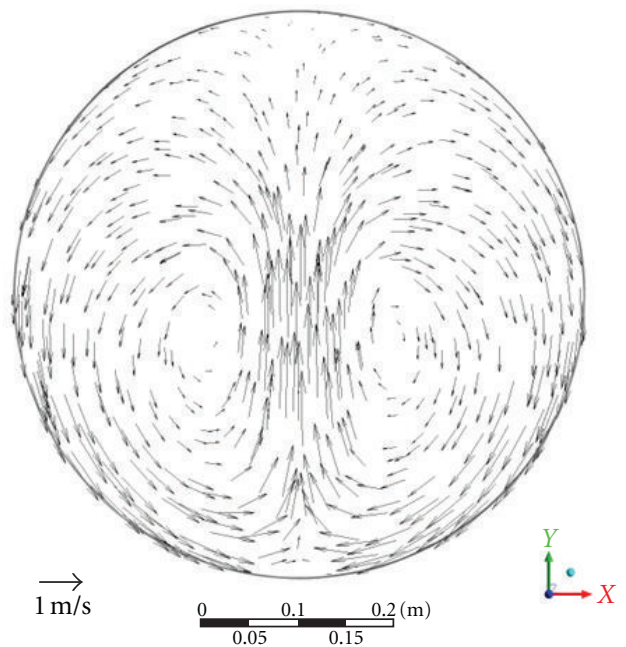

(c) $L_{d}=2 D$

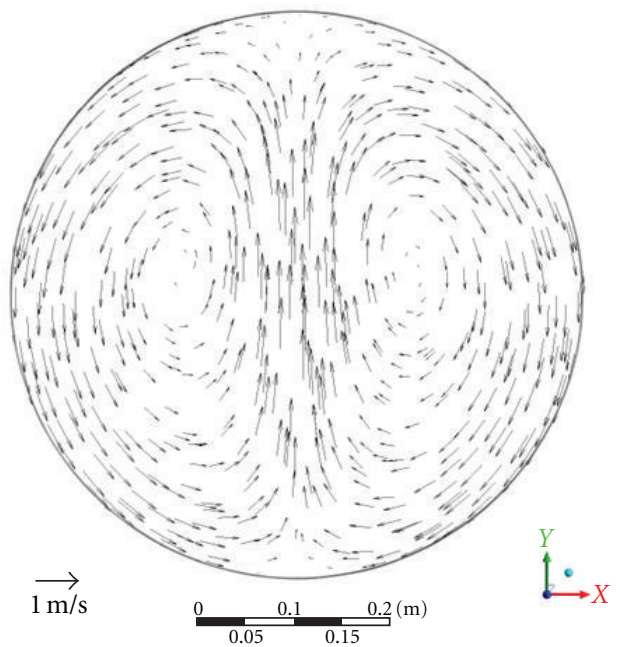

(e) $L_{d}=4 D$

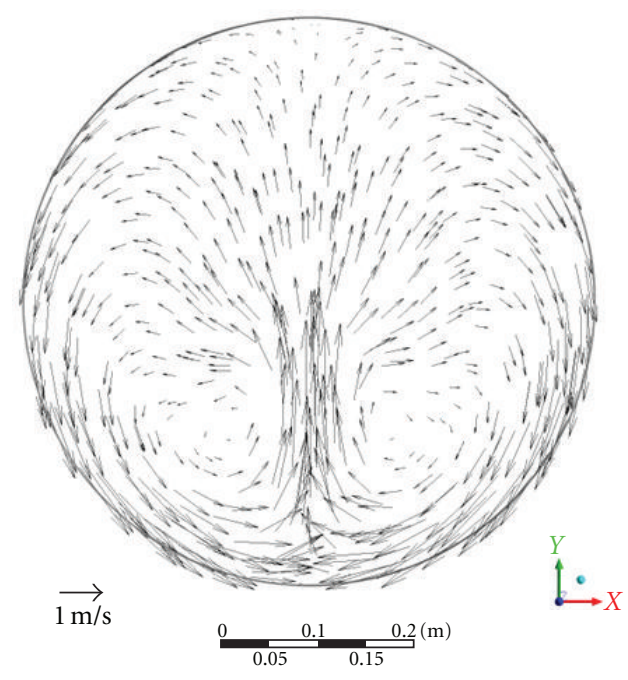

(b) $L_{d}=1 D$

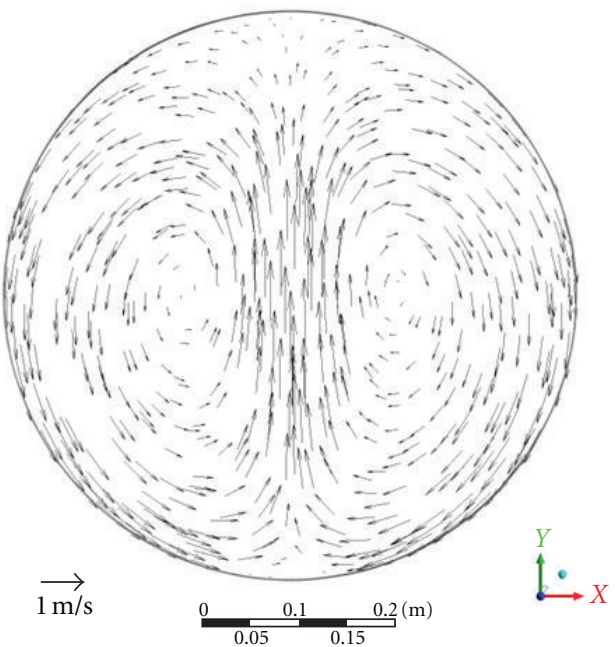

(d) $L_{d}=3 D$

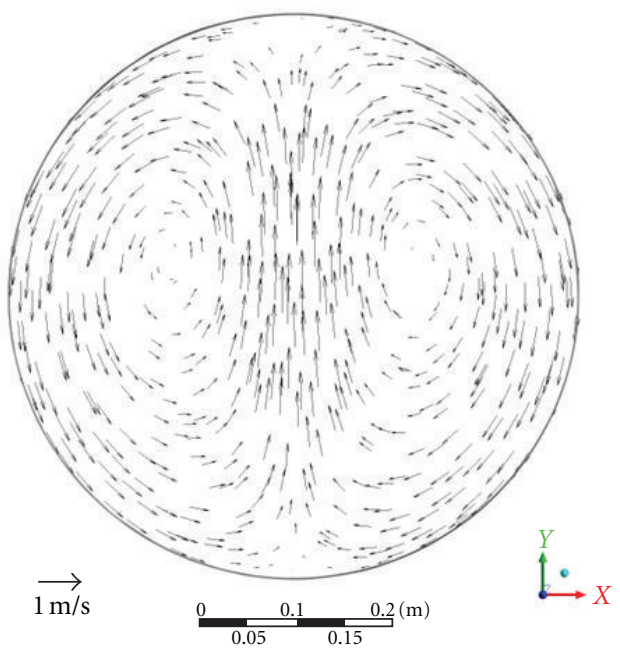

(f) $L_{d}=5 D$

Figure 13: Patterns of secondary flow on each cross-section from 0 to $5 D$ downstream of a single elbow $\left(\operatorname{Re}=5 \times 10^{6}\right)$. Bottom and top show the inner and outer elbow, respectively. 


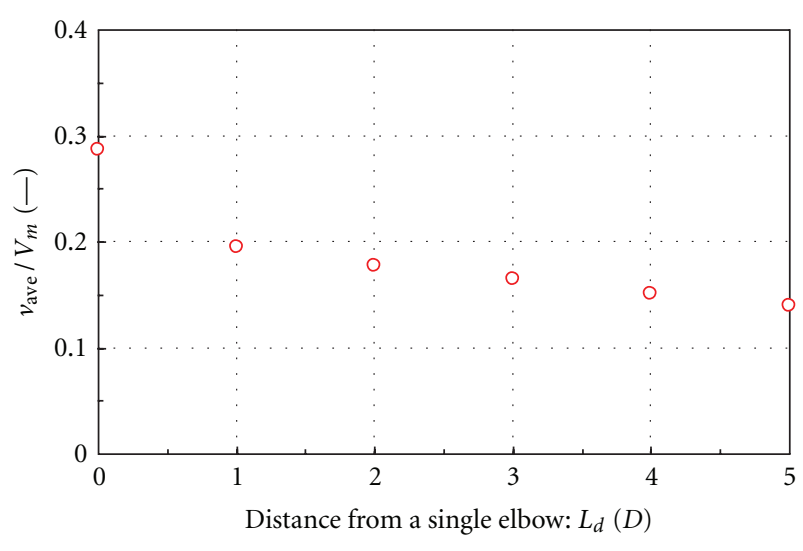

Figure 14: Averaged absolute velocities projected on each crosssection versus distance from the elbow.

TABLE 1: Specifications of CFD analysis.

\begin{tabular}{lc}
\hline Viscous model & SST $\kappa-\omega$ \\
Wall function & Use \\
Time & Steady \\
Pressure-velocity coupling scheme & SIMPLE \\
\hline Spatial discretization & \\
$\quad$ Gradient & Least squares cell-based \\
Pressure & PRESTO! \\
Momentum & Second-order upwind \\
Turbulent kinetic energy & Second-order upwind \\
Specific dissipation rate & Second-order upwind \\
\hline Mesh type & Polyhedra \\
Average mesh size & $0.0254 D$ \\
Number of mesh & $2 \times 10^{6}$ \\
\hline CPU & Intel Xeon $3.2 \mathrm{GHz}$ \\
Number of cores & 3 \\
Number of iterations & 3000 \\
Calculation time per case & $7 \mathrm{~h}$ \\
\hline
\end{tabular}

transmission angle, in other words the ultrasound beam path, would also change in water and the UVP method measures the velocity component along the beam direction. The effects of an incident angle on the accuracy of the UVP method for flow rate measurements were investigated considering longitudinal, shear vertical, and Lamb waves emerging in the pipe wall [13]. However, in power plants, since many carbon steel pipes are used, within which water usually flows, the transmission angle was expected to be within a few degrees difference of $19.4^{\circ}$. Therefore, the transmission angle in this paper was set at $19.4^{\circ}$ as a representative example.

A Shear Stress Transport (SST) $\kappa-\omega$ based model [16] was employed for the turbulent model in this paper, because the SST $\kappa-\omega$ model is described as a better turbulent model than $\kappa-\varepsilon$ models to estimate separation and reattachment points in fully turbulent pipe flow with a sudden expansion or diffuser [17, 18]. Moreover, velocity profiles obtained downstream of an elbow were expected to be influenced by vortexes generated in an elbow. The $\kappa-\omega$ based SST model accounted for the transport of the turbulent shear stress. For free shear flows, the SST model was identical to the $\kappa$ $\varepsilon$ model, with the convergence behavior of the $\kappa-\omega$ model often similar to that of the $\kappa-\varepsilon$ model. This is because the zonal $\kappa-\omega$ models included a blending function in the near wall region as a function of wall distance; an additional equation was solved to compute the wall distance at the start of simulations. The steady flow condition considering gravity and the secondary order upwind for spatial discretization was adopted. The Reynolds number was set at $5 \times 10^{6}$ based on the pipe diameter and the bulk fluid velocity.

The inflow boundary condition for each case consisted of uniform velocity components, while the outflow boundary condition was used for the flow exit model. At the pipe wall, the no-slip condition was applied to the velocity components.

\subsection{Results}

4.2.1. Patterns of Secondary Flow on Each Cross-Section. The computed velocity distributions projected on each crosssection plane from 0 to $5 D$ downstream of a single elbow are shown in Figure 13. It is well known that flow through a pipe elbow results in velocity directions from the inner to the outer wall. This figure shows strong vertical asymmetry due to the pressure gradient and the strong secondary flow generated in the elbow. As seen in this figure, the secondary flows changed significantly between 0 and $1 D$ downstream of the elbow, while two large vortexes emerged after $1 D$, which moved gradually outward from the elbow, increasing the streamwise distance from the same. These secondary flow patterns were expected to reduce the precision of flow rate measurements, because the velocity component along the ultrasound beam path varied depending on the circumferential or streamwise position of the transducer.

To estimate the decrease ratio of secondary flows depending on the distance from the elbow, averaged absolute velocities projected on one cross-section plane and normalized using the bulk fluid velocity were employed. These averaged velocities are shown in Figure 14 with the distance from the elbow on the abscissa. Since the decrease ratio between 0 and $1 D$ is significant, numerous transducers were thought to be required for accurate flow rate metering at $1 D$.

4.2.2. Velocity Profiles along the Ultrasonic Beam Path. Velocity profiles at 1 and $5 D$ along the ultrasonic beam path are illustrated in Figures 15 and 16. As shown in Figure 15(a), the velocity profile at $\phi=90^{\circ}$ varied strongly in the far field, because these velocities were affected by the significant secondary flow between two vortexes, as shown in Figure 13(b). The velocity profile at $\phi=270^{\circ}$ formed a different shape compared with $\phi=90^{\circ}$, since most velocities along the vertical line traversing the center of the pipe in Figure 13(b) pointed upward. Conversely, where the transducer was mounted at $\phi=0$ or $180^{\circ}$, these velocity profiles were almost the same due to the lateral symmetric flow. The velocity profile fluctuation obtained at $5 D$, as shown in Figure 16, was smaller than those at $1 D$, 


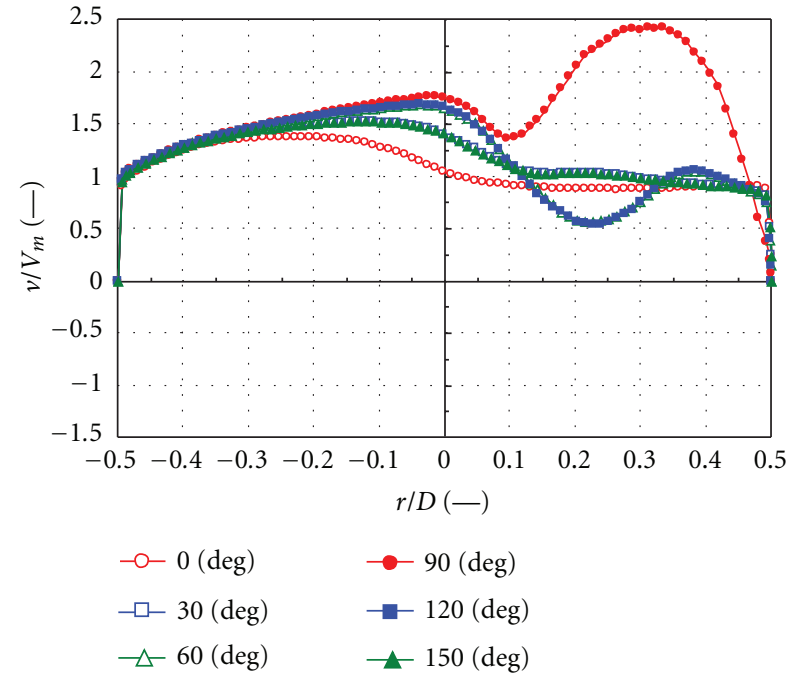

(a) $\Phi=0-150^{\circ}$

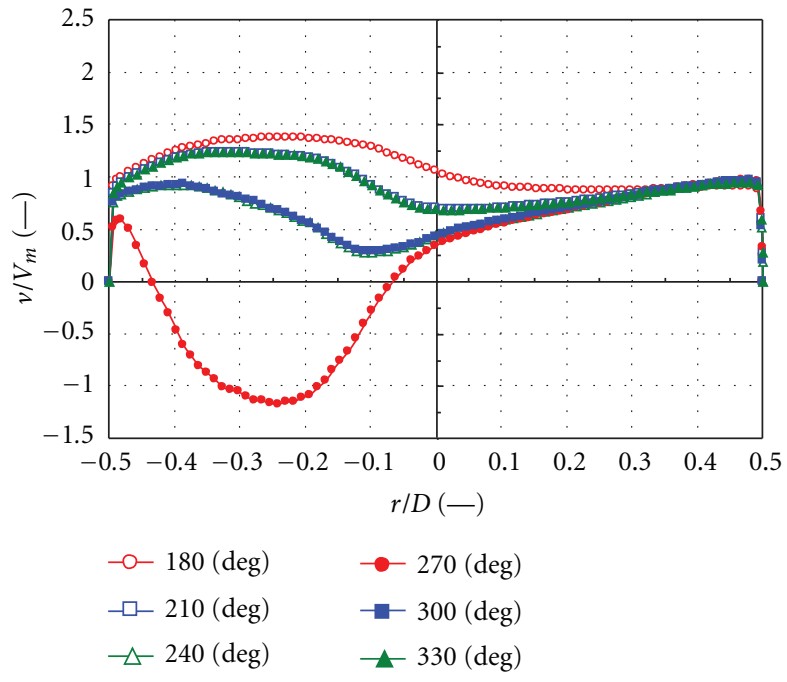

(b) $\Phi=180-330^{\circ}$

FIGURE 15: Velocity profiles along the ultrasonic beam path $\left(\operatorname{Re}=5 \times 10^{6}, L_{d}=1 D\right)$.

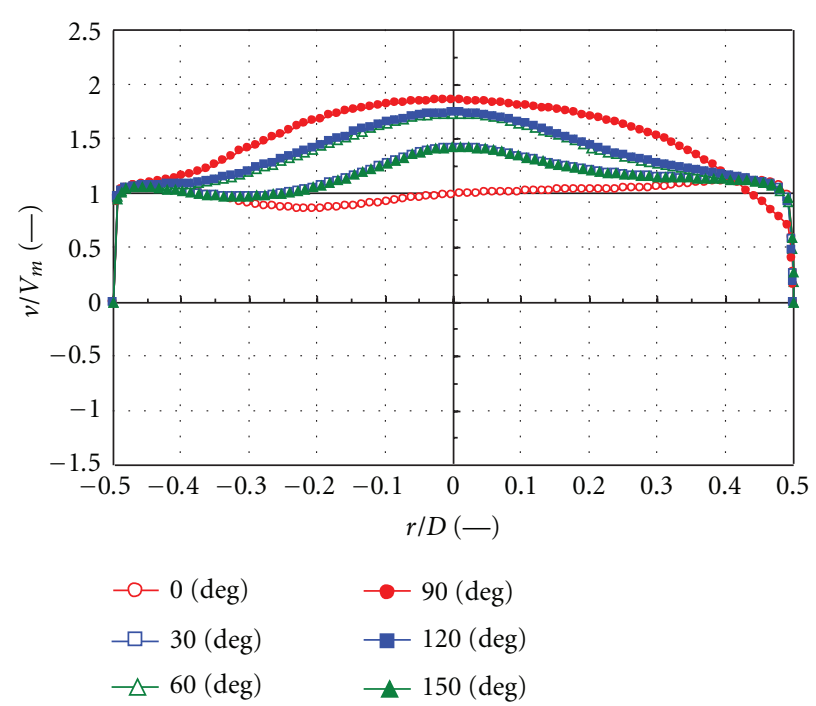

(a) $\Phi=0--150^{\circ}$

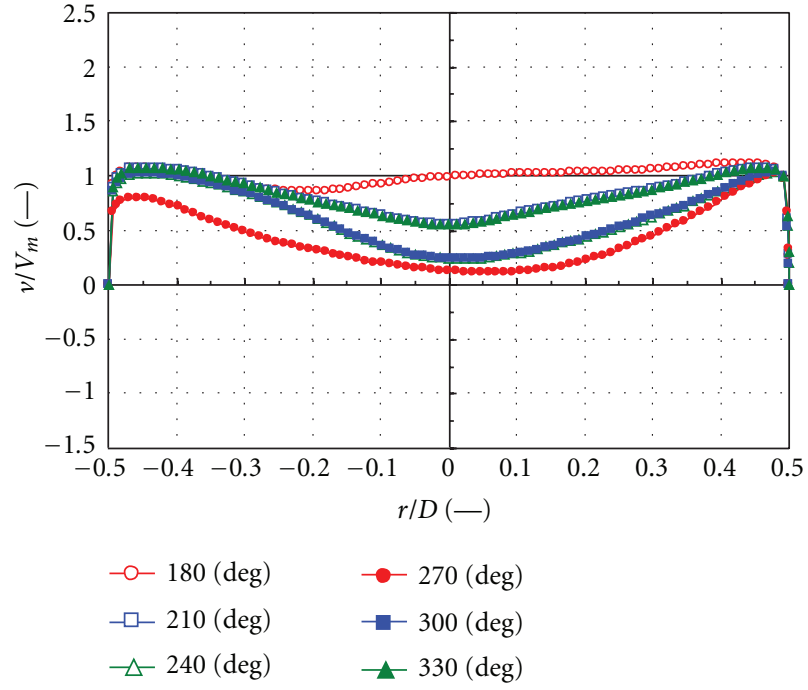

(b) $\Phi=180--330^{\circ}$

FIGURE 16: Velocity profiles along the ultrasonic beam path $\left(\operatorname{Re}=5 \times 10^{6}, L_{d}=5 D\right)$.

because the velocity of the secondary flow between two vortexes was also smaller, as illustrated in Figure 13(f).

In addition, the velocity profiles read out at $\phi=0$ and $180^{\circ}$ resembled the bulk fluid velocity of the pipe flow due to the week secondary flow in a horizontal direction. This result shows the capability to improve the accuracy of flow rate measurement when transducers are located at $\phi=0$ or $180^{\circ}$ downstream of a single elbow, while more quantitative studies should be also conducted.

\subsubsection{Velocity Profile along the Measuring Circle. Figure 17} shows velocity profiles along each measuring circle at $1 D$. The horizontal axis is the transducer circumferential position and the vertical axis is the time-averaged velocity normalized by the mean velocity. The velocity profiles fluctuated locally at $\phi=90$ and $270^{\circ}$, while high frequency fluctuation emerged at $\phi=90^{\circ}$ due to the vertexes shown in Figure 13(b). Conversely, at $5 D$ (see in Figure 18) the frequency declined due to the decreasing vortexes effect.

The flow conditions formed in these simulations were expected to be asymmetrical along a line through $\phi=90$ and $270^{\circ}$ with secondary flows. This is because when focusing on the case of $r / D=-0.3$ and +0.3 in Figures 17 and 18, $V_{\alpha_{-} \text {UVP }}$ (obtained at $\phi=90^{\circ}$ and $r / D=-0.3$ ) differed significantly from $V_{\beta_{-} \text {UVP }}$ (obtained at $\phi=270^{\circ}$ and $r / D=$ -0.3 ) and also from $V_{\gamma_{-} \text {UVP }}$ (obtained at $\phi=270^{\circ}$ and $r / D=+0.3)$. Conversely, the flow conditions were expected to show symmetric flow along a line through $\phi=0$ and 


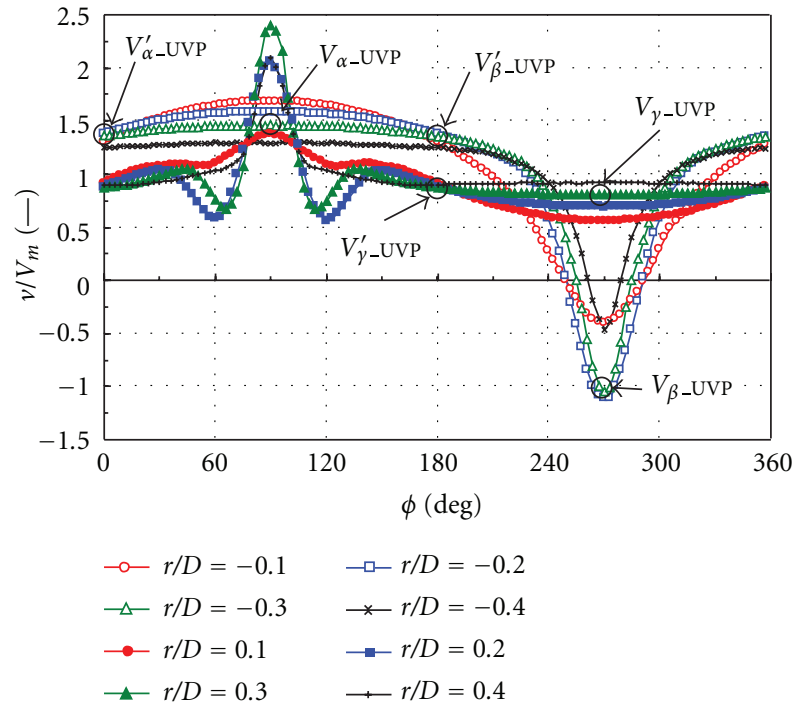

Figure 17: Velocity profiles along the measuring circle $(\mathrm{Re}=5 \times$ $\left.10^{6}, L_{d}=1 D\right)$.

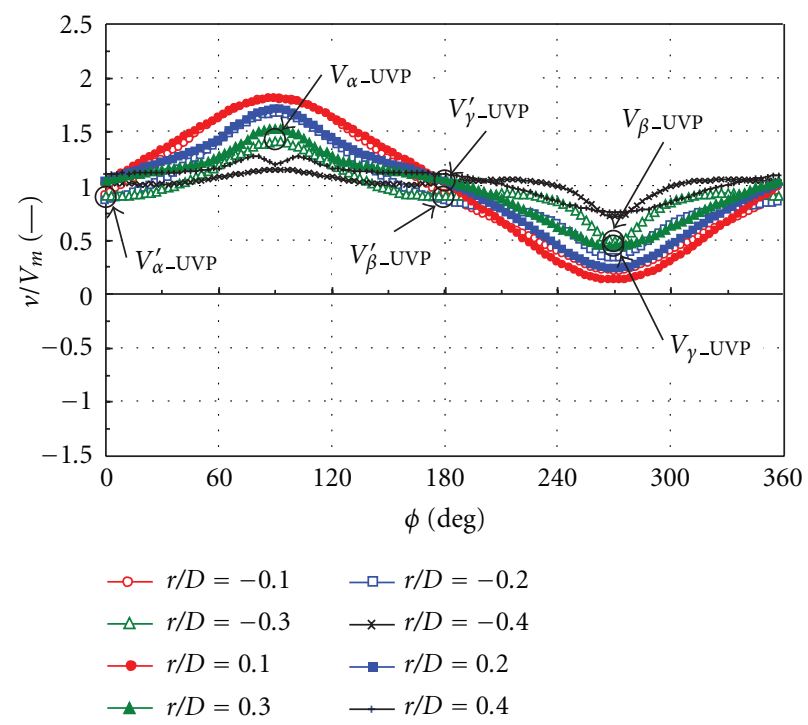

FIGURE 18: Velocity profiles along the measuring circle $(\mathrm{Re}=5 \times$ $\left.10^{6}, L_{d}=5 D\right)$.

$180^{\circ}$ with secondary flows. This is because, $V_{\alpha_{-} \text {UVP }}^{\prime}$ (obtained at $\phi=0^{\circ}$ and $\left.r / D=-0.3\right)$ is almost the same as $V_{\beta_{-} \mathrm{UVP}}^{\prime}$ (obtained at $\phi=180^{\circ}$ and $r / D=-0.3$ ) and differs from $V_{\gamma_{\text {_UVP }}}^{\prime}$ (obtained at $\phi=180^{\circ}$ and $r / D=+0.3$ ). These results show a different tendency of velocity profile compared with the experimental result, due to the difference in Reynolds number and streamwise measuring location.

4.2.4. FFT Analysis of the Velocity Profile along a Measuring Circle. The results of FFT analysis using velocity profiles read out along the measuring circle are illustrated in Figures 19 and 20. Maximum values emerged in which the wave number was approximately $N_{w}=1$, and other local

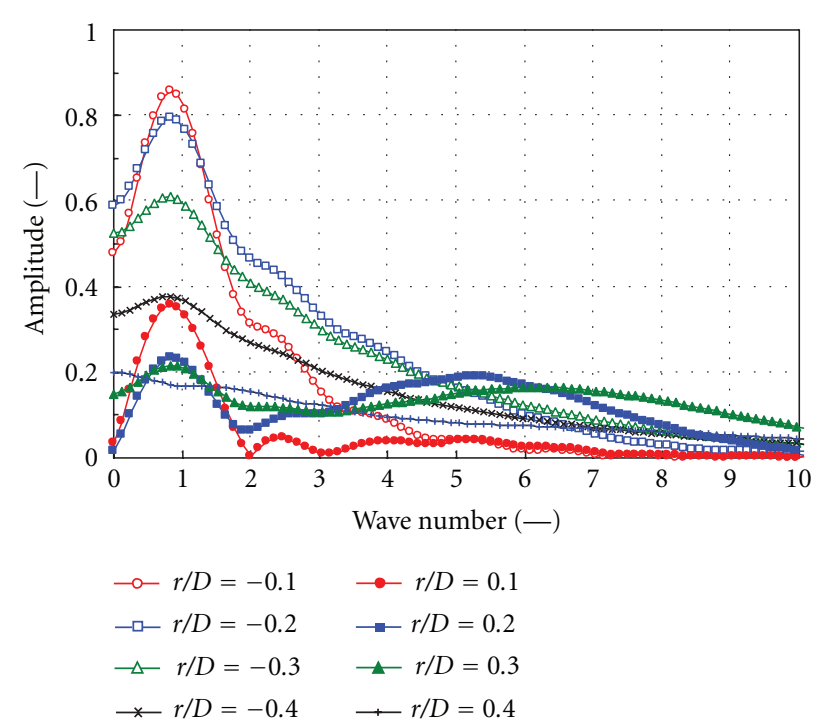

FIGURE 19: Fourier amplitude of the velocity profile along the measuring circle $\left(\operatorname{Re}=5 \times 10^{6}, L_{d}=1 D\right)$.

maximum values appeared between $N_{w}=5$ and 7 for the $1 D$ case (see Figure 19). In addition, the Fourier amplitudes declined remarkably at over $N_{w}=10$, and it is expected that approximately twenty transducers will be required to measure the flow rate accurately, considering the sampling theorem. In the case of $5 D$ shown in Figure 20, the maximum values also appear approximately at $N_{w}=1$ for both 1 and $5 D$ due to the strong secondary flow toward the outer side of the elbow. Employing eight transducers for accurate flow rate metering is expected to be sufficient considering the Fourier amplitudes decreasing completely over $N_{w}=4$.

4.2.5. Flow Rate Considering the Number of Transducers. The flow rate was calculated integrating a velocity profile obtained along a measuring line. When using multiple transducers, these were located on a pipe wall with a constant circumferential interval, and the velocity profiles they obtained were used to calculate the flow rate. Additionally, to investigate the effect of circumferential transducer position $\phi$ on the flow rate measurement error, the transducer position was varied by $3^{\circ}$ and the flow rate was calculated at the each position. Figures 21 and 22 plot both maximum and minimum flow rate errors at 1 and $5 D$ versus the number of transducers for $\operatorname{Re}=5 \times 10^{6}$.

As shown in Figure 21, these maximum and minimum flow rate errors converged when the number of transducers was approximately twenty. This result is consistent with the estimated number of transducers using FFT analysis. The converted values of flow rate errors are approximately +5 and $-6 \%$ for the near and far field, respectively. This is because the patterns of secondary flows, as shown in Figures 13(a) and 13(b), changed significantly depending on the distance from the elbow and the velocity measuring points in the near and far fields were located at different streamwise positions. In the case at $5 D$ (see in Figure 22), these flow rate errors 


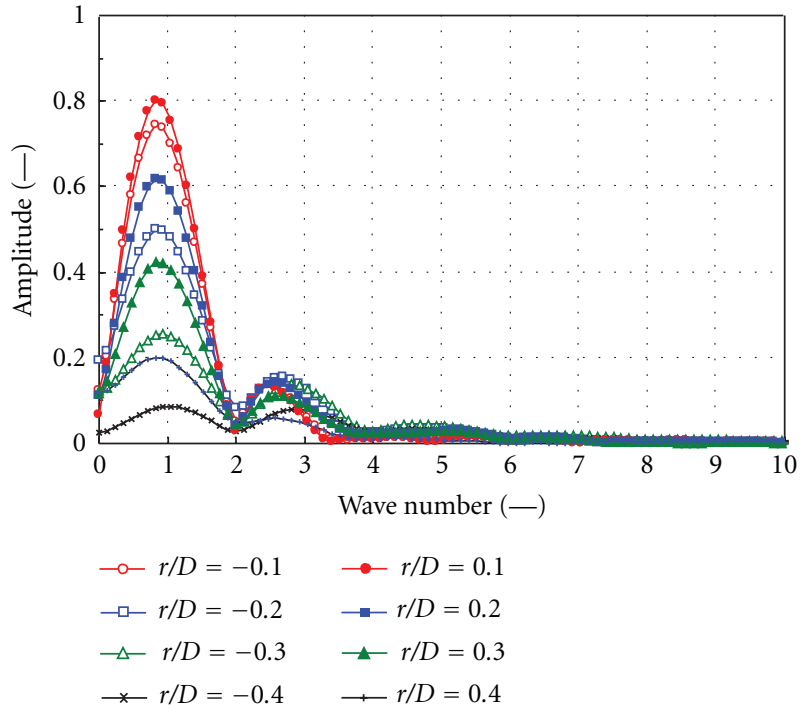

Figure 20: Fourier amplitude of the velocity profile along the measuring circle $\left(\operatorname{Re}=5 \times 10^{6}, L_{d}=5 D\right)$.

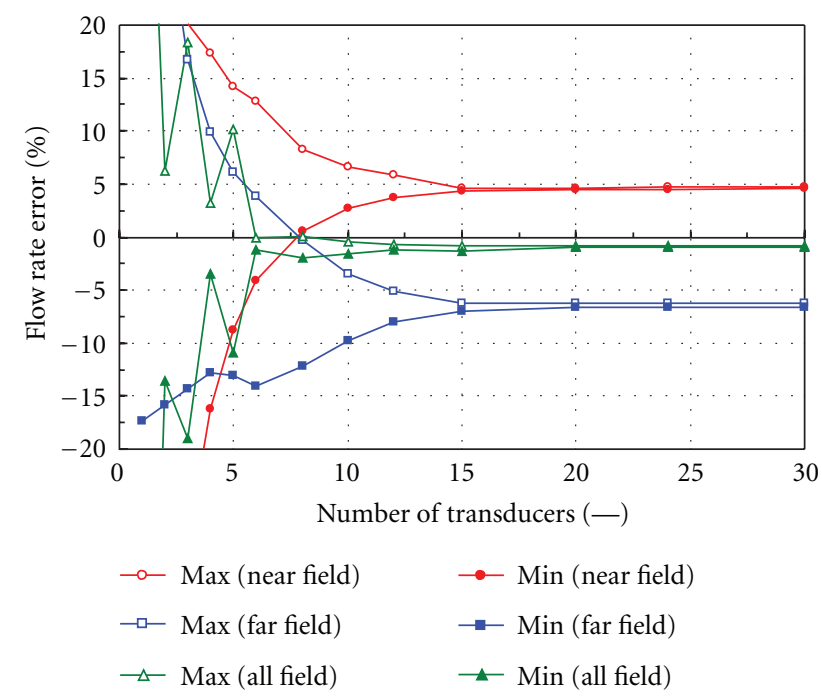

FIGURE 21: Flow rate errors versus the number of transducers $(\mathrm{Re}=$ $\left.5 \times 10^{6}, L_{d}=1 D\right)$.

converged for eight transducers. This result is also consistent with the estimated number of transducers using FFT analysis. An accurate flow rate could be measured at $5 D$ because there was little change in the patterns of secondary flow in a streamwise direction.

\section{Conclusion}

This paper presents a new estimation method to determine the optimal number of transducers using UVP to measure flow rate accurately downstream of a single elbow. The new estimation method employs a wave number of velocity profile fluctuations along a circle on a cross-section of a pipe using FFT. The optimal number of transducers was estimated

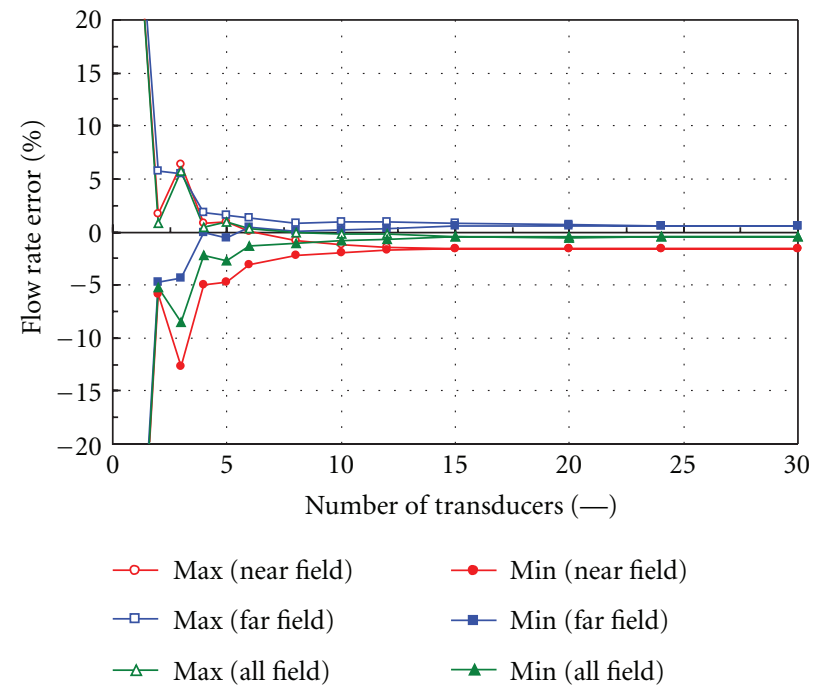

FIGURE 22: Flow rate errors versus the number of transducers $(\mathrm{Re}=$ $\left.5 \times 10^{6}, L_{d}=5 D\right)$.

based on the sampling theorem. To evaluate this method, a preliminary experiment and numerical simulations using CFD were conducted. The evaluation regions of velocity profiles were located at $3 D$ for the experiment, and 1 and $5 D$ for the simulations downstream of the elbow, respectively. Reynolds numbers for the experiment and simulations were set at $4 \times 10^{4}$ and $5 \times 10^{6}$, respectively. These results indicate the flow rate errors converge at the number of transducers which was estimated using the wave number and sampling theorem.

\section{Symbols}

$D: \quad$ Inner pipe diameter

$r$ : Radius position in a pipe

$V_{m}:$ Bulk fluid velocity

$V_{\text {TX }}$ : Velocity component along the ultrasound beam path

$V_{\text {axial }}$ : Velocity component in a streamwise direction

$\theta: \quad$ Incidence or refraction angle of ultrasound

$c_{f}$ : Sound speed in fluid

$f_{0}$ : Frequency of ultrasound emitted from transducer

$f_{d}$ : Doppler shift frequency

$t_{f}$ : Traveling time of the ultrasound pulse

$L_{f}$ : Distance from transducer to particle

$L_{d}$ : Streamwise distance from the outlet plane of a single elbow

A: Amplitude of the ultrasound pulse

$\lambda, \mu$ : Lame constant

$\phi: \quad$ Circumferential angle

$N_{w}$ : Wave number of the velocity profile in a measuring circle. 


\section{Subscripts}

$a, b, c, d$ : Velocity component

$0 \sim 2$ : Medium: 0 is water, 1 is carbon steel, and 2 is water

$l$ : $\quad$ Longitudinal wave

s: $\quad$ Shear vertical wave

$i$ : $\quad$ Incidence

$t$ : Transmission.

\section{References}

[1] M. Mori, Y. Takeda, T. Taishi, N. Furuichi, M. Aritomi, and H. Kikura, "Development of a novel flow metering system using ultrasonic velocity profile measurement," Experiments in Fluids, vol. 32, no. 2, pp. 153-160, 2002.

[2] K. Tezuka, M. Mori, T. Suzuki, and Y. Takeda, "Calibration tests of pulse-Doppler flow meter at national standard loops," Flow Measurement and Instrumentation, vol. 19, no. 3-4, pp. 181-187, 2008.

[3] K. Tezuka, M. Mori, T. Suzuki, M. Aritomi, H. Kikura, and Y. Takeda, "Assessment of effects of pipe surface roughness and pipe elbows on the accuracy of meter factors using the ultrasonic pulse doppler method," Journal of Nuclear Science and Technology, vol. 45, no. 4, pp. 304-312, 2008.

[4] S. Wada, H. Kikura, M. Aritomi, M. Mori, and Y. Takeda, "Development of pulse ultrasonic doppler method for flow rate measurement in power plant multilines flow rate measurement on metal pipe," Journal of Nuclear Science and Technology, vol. 41, no. 3, pp. 339-346, 2004.

[5] S. A. Berger, L. Talbot, and L. S. Yao, "Flow in curved pipes," Annual Review of Fluid Mechanics, vol. 15, pp. 461-512, 1983.

[6] M. Anwer and R. M. C. So, "Swirling turbulent flow through a curved pipe part I: effect of swirl and bend curvature," Experiments in Fluids, vol. 14, no. 1-2, pp. 85-96, 1993.

[7] S. A. Slimon and D. W. Davis, "Computational prediction of secondary flows in complex piping systems," American Society of Mechanical Engineers Fluids Engineering Division, vol. 196, pp. 393-403, 1994.

[8] M. J. Tunstall and J. K. Harvey, "On the effect of a sharp bend in a fully developed turbulent pipe flow," Journal of Fluid Mechanics, vol. 34, p. 535, 1968.

[9] G. E. Mattingly and T. T. Yen, "Secondary flow effects due to several elbow configurations," American Society of Mechanical Engineers Fluids Engineering Division, vol. 95, pp. 5-9, 1990.

[10] Y. Takeda, "Velocity profile measurement by ultrasound Doppler shift method," International Journal of Heat and Fluid Flow, vol. 7, no. 4, pp. 313-318, 1986.

[11] Y. Takeda, "Measurement of velocity profile of mercury flow by ultrasound Doppler shift method," Nuclear Technology, vol. 79, no. 1, pp. 120-124, 1987.

[12] K. Tezuka, S. Wada, M. Mori, H. Kikura, and M. Aritomi, "Analysis of ultrasound propagation in a steel pipe using Ultrasonic Pulse Doppler method," Thermal Science \& Engineering, vol. 16, no. 3, pp. 95-104, 2008 (Japanese).

[13] Y. Inoue, H. Kikura, H. Murakawa, M. Aritomi, and M. Mori, "A study of ultrasonic propagation for ultrasonic flow rate measurement," Flow Measurement and Instrumentation, vol. 19, no. 3-4, pp. 223-232, 2008.

[14] T. Shiraishi, H. Watakabe, H. Sago, and H. Yamano, "Pressure fluctuation characteristics of the short-radius elbow pipe for FBR in the postcritical Reynolds regime," Journal of Fluid Science and Technology, vol. 4, no. 2, pp. 430-441, 2009.
[15] W. Treenuson, N. Tsuzuki, H. Kikura, M. Aritomi, S. Wada, and K. Tezuka, "Development of flow rate measurement in the bent pipe using ultrasonic velocity profile method," in Proceedings of the 9th International Topical Meeting on Nuclear Thermal-Hydraulics, Operation and Safety (NUTHOS9), N9P0293, Kaohsiung, Taiwan, September 2012.

[16] F. R. Menter, "Zonal two equation $\kappa-\omega$ turbulence models for aerodynamics flows," in AIAA Paper 93-2906, 1993.

[17] J. E. Bardina, P. G. Huang, and T. J. Coakley, "Turbulence modeling, validation, testing and development," NASA Technical Memorandum 110446, 1997.

[18] J. W. Baughn, M. A. Hoffman, R. K. Takahashi, and B. E. Launder, "Local heat transfer downstream of an abrupt expansion in a circular channel with constant wall heat flux," Journal of Heat Transfer, vol. 106, no. 4, pp. 789-796, 1984. 

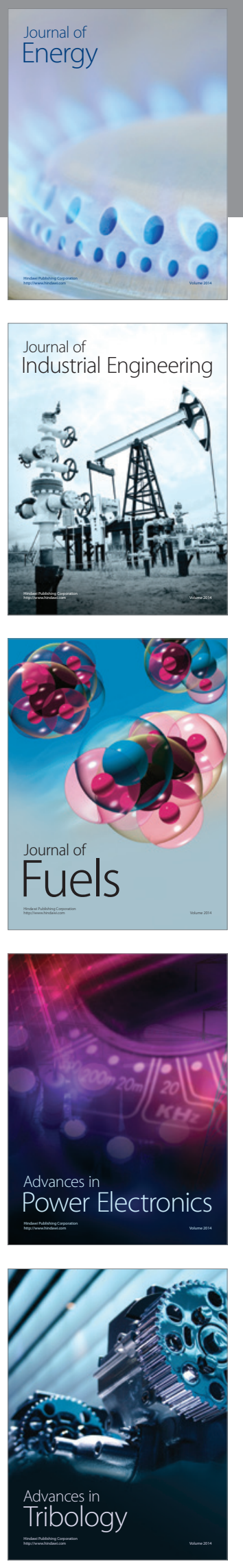
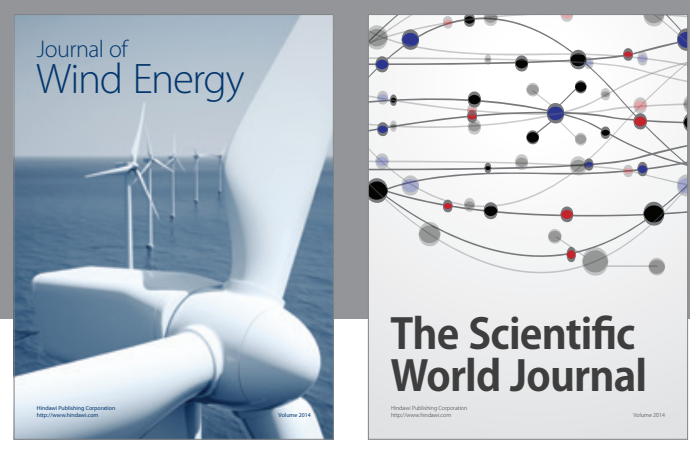

The Scientific World Journal

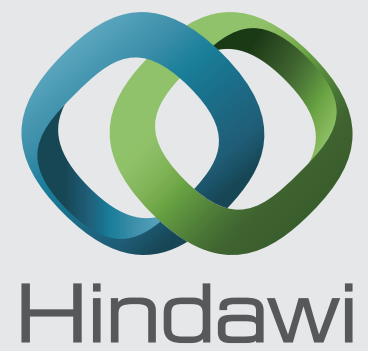

Submit your manuscripts at http://www.hindawi.com
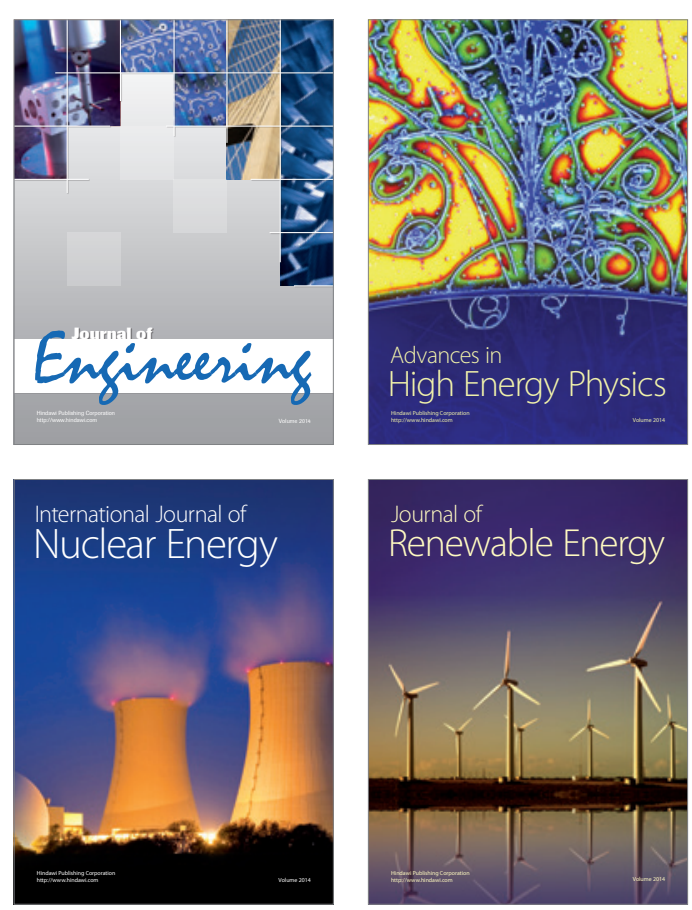

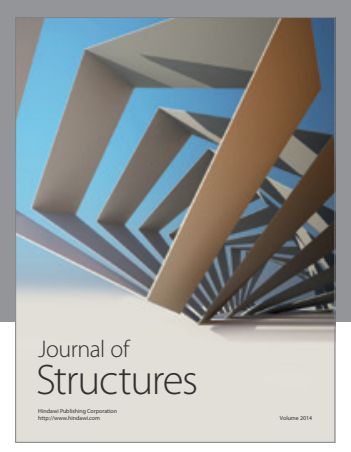

Rotating
Mechinery
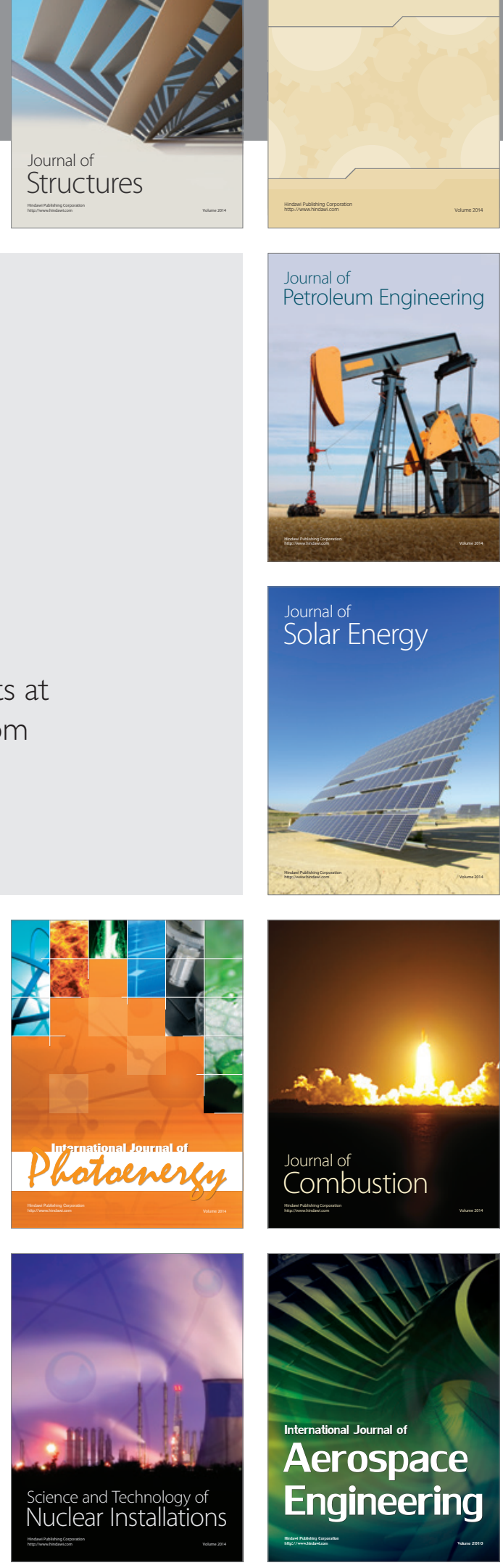